\title{
The Management of Hepatitis B Flare in Pregnancy
}

\author{
Arina Mana Sikana ${ }^{a^{*}}$, Husin Thamrin ${ }^{\mathrm{b}}$ \\ a arinamanasikana010890@gmail.com \\ ${ }^{a}$ Department of Internal Medicine, Airlangga University-Dr Soetomo General Hospital, Prof. Dr. Moestopo St. No 6 - 8, Surabaya \\ 60286, Indonesia \\ ${ }^{\mathrm{b}}$ Division of Tropical and Infectious Diseases, Department of Internal Medicine, Airlangga University-Dr Soetomo General Hospital, \\ Prof. Dr. Moestopo St. No 6 - 8, Surabaya 60286, Indonesia
}

\begin{abstract}
Background: Hepatitis B infection during pregnancy is still a unique problem for the mother and her fetus. These include the effects of hepatitis B on maternal and fetal health, the effects of pregnancy on hepatitis B infection in pregnancy, treatment of hepatitis B during pregnancy and prevention of mother-to-child transmission.

Case description: A young woman was referred for jaundice in pregnancy. Physical examination revealed icteric sclera in both eyes. Laboratory tests show increased liver function as well as bilirubin. for testing for hepatitis $\mathrm{B}$ reactive. The patient received lamivudine therapy and cesarean section therapy.

Conclusion: Management and treatment of chronic hepatitis B is difficult because of the variable course of the disease. It is even more complex in pregnant patients. Joint decision-making between physicians and female patients regarding the timing of initiation of antiviral therapy is critical to improving outcomes. Optimal initiation of antiviral therapy in the general population depends on the histologic degree of inflammation and fibrosis. We now know that post-partum HBIG and vaccination for infants born to chronically infected mothers can be ineffective in preventing MTCT to regulate increased maternal HBV viremia.
\end{abstract}

Keywords: Hepatitis B Flare; Management; Lamivudine; MTCT

\section{Introduction}

HBV infection in pregnancy is still an important global health problem. More than $50 \%$ of the 350 million hepatitis B carriers have perinatal infection and the risk of chronic infection progression is inversely related to age at infection (Alter et al., 2000). The risk is as much as $90 \%$ of exposure in infants without vaccination, while the risk is lower by $20-30 \%$ in infants exposed in childhood (Borgia et al., 2012). Significantly, women of reproductive age with chronic hepatitis B infection are still the source of HBV transmission worldwide. Thus, hepatitis B therapy during pregnancy is very important in interfering with perinatal HBV transmission.

Perinatal or childhood transmission of HBV generally leads to chronic hepatitis which causes necroinflammation and fibrosis and results in a higher risk of developing cirrhosis and hepatocellular carcinoma. More than $50 \%$ of chronic hepatitis B carriers in endemic areas acquire the infection perinatally. In the absence of prevention, infants born to $\mathrm{HBeAg}$ positive mothers have a 40-90\% risk of getting chronic hepatitis B through vertical transmission (Balsano et al., 2016). The main risk factor for failure of immunoprophylaxis is the amount of maternal HBV DNA during the third trimester. Therapy nucleoside or nucleotide analogue is required in highly viremic women of serum HBV DNA between 28 and 32 weeks (Elefsiniotis et al., 2015).

Therefore, screening and management of chronic hepatitis in pregnancy is very important to prevent mother-to-child transmission (vertical transmission) to reduce the burden of chronic hepatitis B in the world.

\section{Case Description}

A pregnant woman, Mrs. C, 28 years old, lives in Sidoarjo, works as a housewife. Came to the hepato poly on February 18, 2020 with complaints of both yellow eyes since 2 months ago. Referral patient from RSUD Sidoarjo with G2P1A0 gravid 36 weeks after one cesarean section with hepatitis B with jaundice pregnancy. The patient admitted that both his eyes were yellow since 2 months ago, yellow was also recognized on the skin of the hands and feet. The patient noticed that his eyes were yellow since the pregnancy check-up at the Gunung Anyar Health Center. Then when it was checked for HBsAg +. There are complaints of weakness in the body, no fever and sometimes complaints of itchy skin. The patient also admitted that his urine was thick like tea for the past 2 months. Bowel movement are recognized as normal, there is no change in color or frequency. The patient also admitted that 2 weeks ago he had nausea and vomiting and his stomach felt tight so he was treated for 1 week at the Sidoarjo Hospital. Then the patient was advised to control the Hepato poly, RSUD Dr. Soetomo. The patient had not received hepatitis B therapy from the previous hospital. History of jaundice in previous pregnancies was denied. The patient had no previous history of jaundice, diabetes and hypertension was denied. TB disease is also denied. No family members suffer from a similar disease, namely hepatitis B, no family members suffer from other liver diseases, diabetes or hypertension. The patient is a 
housewife, has a child who is 3 years old. Born by caesarean section on the indication of a large baby. The patient lives with her husband who works as an intercity bus driver. History of taking drugs and herbal medicine was denied. diseases, diabetes or hypertension. The patient is a housewife, has a child who is 3 years old. Born by caesarean section on the indication of a large baby. The patient lives with her husband who works as an intercity bus driver. History of taking drugs and herbal medicine was denied.

From the examination of vital signs, the general condition was sufficient with GCS E4V5M6. BP $120 / 80 \mathrm{mmHg}$, pulse $96 \mathrm{x} / \mathrm{m}$ regular rhythm, lifting strength, RR 18x/m, temperature 36.5 . On examination of the head/neck of the conjunctiva, there was no anemia, icteric sclera in both eyes, neither cyanosis nor dyspnea were found. There was no increase in jugular venous pressure and neck lymph node enlargement.

The results obtained were $\mathrm{Hb} 12.7 \mathrm{~g} / \mathrm{dL}$, wbc 8140/uL, platelets 178000/uL, RBC $4.0 \times 106 / \mathrm{uL}$, PPT/control 13.6/15.4, APTT/control 34.4/31.9, Glucose $99 \mathrm{mg} / \mathrm{dL}$, BUN $5.1 \mathrm{mg} / \mathrm{dL}$, Creatinine $0.4 \mathrm{mg} / \mathrm{dL}$, Albumin $3.4 \mathrm{mg} / \mathrm{dL}$, Direct Bilirubin $6.77 \mathrm{mg} / \mathrm{dL}$, Total Bilirubin $7.8 \mathrm{mg} / \mathrm{dL}$, SGOT 1875 U/L, SGPT 1101 U/L. Sodium $138 \mathrm{mmol} / \mathrm{L}$, Potassium $4.1 \mathrm{mmol} / \mathrm{L}$, Cloride $108 \mathrm{mmol} / \mathrm{L}, \mathrm{HBsAg}$ rapid reactive, IgM anti HAV non reactive, IgG anti HAV non reactive. Complete Urine Examination: BJ 1.025, pH 5.0, Leukocytes +2 , nitrite negative, normal albumin, normal glucose, Ketones +1 , Urobilinogen +1 , Bilirubin +3 , Erythrocytes +1 , Hb negative, cylinder +, bacteria +, Crystal negative , erythrocytes (sediment) 8-10, leukocytes (sediment) 1215. Abdominal ultrasound examination at Sidoarjo Hospital (5 February 2020). Enlarged liver, decreased echoparenchym intensity, V.porta \& V. hepatica are not dilated, IHBD/EHBD widening is not visible, no solid nodules/cysts are seen. Gall bladder: normal size. Thickened walls, no visible stones/ solid nodules/ bile sludge. Pancreas; Normal size, no solid nodule/cyst visible. Ren dextra sinistra: normal size, echocortical intensity did not increase, the apelviocalyceal system was not dilated, no stones/solid nodules/cysts were seen. Buli-buli: even out line, not thickened walls, no visible stones/solid nodules. Uterus: enlarged with fetus, single, alive. Adnexa: no solid nodule/cyst, no endometrial thickening seen. Conclusion: non-obstructive jaundice with hepatomegaly (hepatitis). Supporting examination at Sidoarjo Hospital (7 February 2020). Glucose $116 \mathrm{mg} / \mathrm{dL}$, Direct Bilirubin $5.61 \mathrm{mg} / \mathrm{dL}$, Total Bilirubin $8.24 \mathrm{mg} / \mathrm{dL}$, SGOT $498 \mathrm{U} / \mathrm{L}$, SGPT 581 U/L, Anti HCV non reactive.Based on history, physical examination and investigations, our patient was diagnosed as G2P1A0 gravid 36 weeks with jaundice pregnancy + chronic reactivation hepatitis B. Our patient plans a follow-up examination in the form of HBeAg + HBV DNA, as well as periodic evaluations of SGOT, SGPT, Direct Bilirubin, Total Bilirubin, and APTT and PTT. Examination of UL and urine sediment; GDP, Lipid Profile, Uric Acid.

Initial Therapy for the patient Diet H2 $2100 \mathrm{kcal} /$ day low fat, Inf. D5 1000cc every 24 hours, lamivudine $100 \mathrm{mg}$ every 24 hours po, Ursodeoxycholic acid $250 \mathrm{mg}$ every 8 hours po, Inj. Vit K 1 Ampoules every 8 hours. 20th February 2020 Caesarean section was performed on the patient and a baby boy was born $2800 \mathrm{mg}$, PB $45 \mathrm{~cm}$ and was given HbIg injection and hepatitis B vaccine.

\section{Discussion}

Acute hepatitis B virus (HBV) infection during pregnancy is usually mild and is not associated with increased mortality or is teratogenic. With infection during pregnancy should not immediately to consider termination of pregnancy. However, there have been reports of an increased incidence of low birth weight and premature babies in mothers with hepatitis B infection (Wilkins, 2017).

HBeAg status had the highest impact on vertical hepatitis B transmission. Without prophylaxis, $<10 \%$ of HBeAg negative mothers have a risk of transmitting perinatal HBV infection to their infants but 70 to $90 \%$ if the mothers are $\mathrm{HBeAg}$ positive. $90 \%$ have a chance of being a chronic carrier of $\mathrm{HBV}$ if infected at birth and when chronically infected have $15-25 \%$ die in adulthood of cirrhosis or liver cancer. However, $85-95 \%$ effectively reduce HBV infection from vertical transmission by administering a combination of hepatitis B vaccine and hepatitis B immune when given within 12 hours of delivery (Deep et al., 2016).

The history includes birth, family history of HBV infection and liver cancer, history of smoking, tattoos, injection drugs, transfusions, sexual habits, travel to endemic countries, and work (Gyawali, 2012).

In the anamnesis there was no family history of previous HBV infection, liver cancer, no smoking, tattoos, previous transfusions, and others.

Clinical manifestations of chronic viral hepatitis vary significantly from asymptomatic to manifestations of advanced liver disease (cirrhosis or HCC). Often, patients show vague symptoms such as malaise, fatigue and weakness, abdominal pain, myalgia, arthralgia, nausea, and anorexia (Gyawali, 2012).

The patient had symptoms of both yellow eyes since 2 months ago, fatigue and limb weakness for 1 month. Previously also had a history of nausea and vomiting. And the patient also admitted that the color of his urine was thick since 2 months ago. 


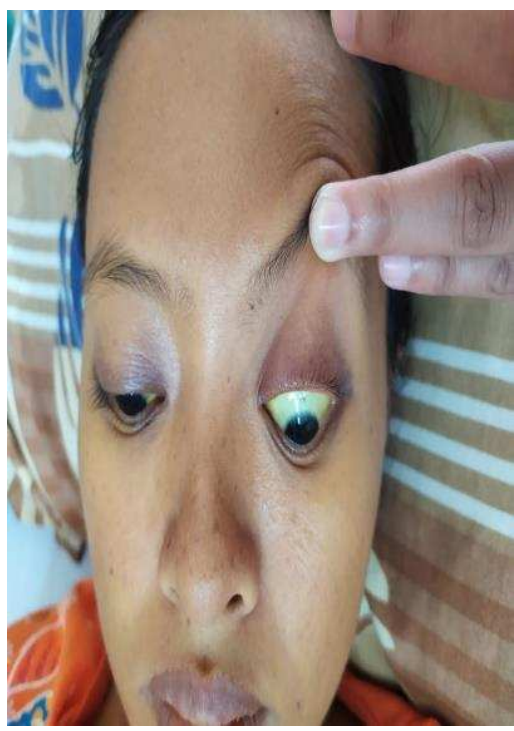

Figure 1. Clinical picture of the patient

Physical examination findings of chronic viral hepatitis vary according to the stage of disease at diagnosis. In the early phase, the patient may show no abnormalities on physical examination. Physical examination findings of cirrhosis may include jaundice, parotid gland enlargement, gynecomastia, ascites, abdominal collateral circulation, peripheral edema, telangiectasia, muscle wasting, palmar erythema, and altered mental status; These findings are not specific for a particular etiology of liver disease (Gyawali, 2012).

In this patient, both icterus eye sclera were found, both palms looked yellow, no ascites was found, collateral circulation in the abdomen and palmar erythema was not found.

Chronic HBV infection occurs 10-15\% in infected adults, 30-50\% in children infected before the age of 6 years and $80-90 \%$ in infants. asymptomatic, normal or enlarged liver, and mild or normal increase in liver function are usually signs of chronic infection in a person (Bartholomew, M.L. 2017).

On supporting examination in the form of abdominal ultrasound, this patient found an enlarged liver and on laboratory examination there was an increase in ALT 1101 and AST 1875.

HBsAg should be tested in all pregnant women at the first prenatal visit even if they have been vaccinated or tested previously. To identify it with a complete history and physical examination. The 2015 American Congress of Obstetrics and Gynecology (ACOG) and CDC developed a screening and management algorithm for $\mathrm{HBV}$ in pregnancy. If $\mathrm{HBsAg}$ is positive, $\mathrm{HBeAg}$ testing should be performed to assess infectivity and AST, ALT) and HBV DNA (Bartholomew, M.L. 2017).

In this patient with HBsAg positive but in waiting for the results of HBV DNA and evaluation of fibrosis, consideration of $\mathrm{HBV}$ reactivation that occurred in the patient and the risk of perinatal transmission so that antiviral therapy was started with lamivudine $1 \times 100 \mathrm{mg}$.

Chronic hepatitis B progresses through four phases. Variations in phase duration and frequency fluctuations between phases of infection can make it difficult to characterize a patient's status.

This patient was found to be HBsAg positive for the first time during his current pregnancy, in February 2020. HBeAg was non-reactive. HBV DNA2.82x $104 \mathrm{Log} 4.45 \mathrm{IU} / \mathrm{mL}$. There was an increase in ALT 1101 and AST 1875. Liver biopsy was not performed on the grounds of an invasive procedure that would endanger the pregnancy. So we diagnosed this patient with Jaundice on pregnancy ec Hepatitis B reactivation of GIIP2A0 gravid 36 weeks THIU (Single, Live, Intrauterine). And this patient is an indication for treatment.

To reduce the risk of perinatal transmission in gravida with higher HBV DNA by using antivirals during the third trimester (Pan et al., 2016).

Therapeutic goals in Hepatitis B patients include (European Association for the Study of the Liver, 2017):

- Long-term suppression of HBV DNA levels is the primary goal of all current therapeutic strategies.

- Induction of HBeAg loss, with or without anti-HBe seroconversion, in HBeAg positive chronic Hepatitis B patients is a significant end point, as it often indicates partial immune control of chronic HBV infection.

- A biochemical response defined as normalization of ALT should be considered as a goal of adjunct therapy, which is generally seen in patients with long-term suppression of HBV replication.

- HBsAg loss, with or without anti-HBs seroconversion, is the optimal goal, as it indicates (complete) suppression of HBV replication and viral protein expression.

This patient was found to be HBsAg positive, the day before the cesarean section was given therapy with lamivudine 1x $100 \mathrm{mg}$ and then it was recommended to check for HBV DNA and HBeAg. The results of 
HBV DNA 2.82x $104 \mathrm{Log} 4.45 \mathrm{IU} / \mathrm{mL}$ and non-reactive HBeAg were obtained. And we continued lamivudine therapy until approximately 3 months after delivery by monitoring ALT and HBV DNA at 1, 3 and 6 months of birth.

Initiation of antivirals in highly viremic patients at 28-32 weeks of gestation to reduce MTCT (Mother To Child Transmission) is recommend by The American College of Gastroenterology (ACG) and AASLD. First line therapy is tenofovir and telbivudine (Ayoub and Cohen, 2016).

Han et al evaluated initiation of lamivudine in the second and third trimesters in women with high viremia and HBeAg positive. There were similarities between mothers who received telbivudine and mothers who received lamivudine and had a high response rate (160/164), and a high ALT normalization rate $(90.2 \%$ vs. 55.88\%). Infants aged 7-12 months after birth were not found to be HBsAg positive in mothers treated with lamivudine compared to the control group (not receiving lamivudine therapy). None of the women treated with lamivudine required discontinuation of treatment because of resistance or side effects. In general, resistance is very rare in short-term therapy (Xu et al., 2009). To avoid the development of resistance and to minimize fetal exposure, lamivudine in the third trimester can be used. if someone is resistant to lamivudine in therapy, it can be replaced with tenovofir (Ayoub and Cohen, 2016).

In this patient, considering that he will receive short-term therapy while waiting for his HBV DNA and $\mathrm{HBeAg}$ examination, the patient has been given an antiviral in the form of lamivudine $1 \mathrm{x} 100 \mathrm{mg}$ while looking at several studies of resistance that rarely occur to short-term therapy and with the patient's social considerations.

According to EASL (2017), in all pregnant women with high HBV DNA levels $(200,000 \mathrm{IU} / \mathrm{ml})$ or HBsAg levels ( $4 \log 10 \mathrm{IU} / \mathrm{ml}$ ), antiviral prophylaxis with TDF (Tenofovir) should be started at week 24-28 of gestation and continued up to 12 weeks after delivery. ALT and HBV DNA levels should be monitored in all HBsAg-positive pregnant women at 1, 3 and 6 months after delivery. In patients with cirrhosis of the liver before pregnancy, antiviral therapy (LAM-Lamivudine, TDF or LdT-Telbivudin) is recommended first, with one of these drugs continuing throughout pregnancy and monitoring should also be performed.

The use of antiviral therapy in pregnancy is slightly different. Due to the inhibition of cell proliferation, interferon is contraindicated in pregnant women. For patients taking interferon, pregnancy can progress after stopping interferon for 6 months. LdT and TDF are FDA category B antiviral drugs for use in pregnancy. After reviewing safety data on LAM in clinical practice LAM was appointed to a category B antiviral drug in pregnancy by the NIH (National Institutes of Health). So, category B antiviral drugs in pregnancy are LAM, LdT and TDF (Tran, 2009).

All HBV clinical practice guidelines recommend prenatal HBsAg screening in pregnancy and postnatal passive-active immunoprophylaxis with HBIG together with a three-dose serial HBV vaccine for infants born to HBsAg+ mothers (Joshi et al., 2020).

The method of delivery in this patient was using the sectio caesarean method by considering the risk of vertical transmission from mother to fetus.

HBsAg, HBeAg and HBV DNA are excreted in breast milk in mothers infected with hepatitis B. According to WHO, there is no increased risk of HBV transmission through breastfeeding even in the absence of immunization. However, breastfeeding should be avoided on sore or bleeding nipples because this will result in the mixing of exudate with breast milk which has the potential to transmit Hepatitis B.

\section{Conclusion}

Based on history, physical examination and investigations, our patient was diagnosed as G2P1A0 gravid 36 weeks with jaundice pregnancy + chronic reactivation hepatitis B.

\section{Acknowledgements}

The authors would like to thank to the deceased patient and his caregivers who allowed this case tobe documented.

\section{References}

Alter, M.J. and Ph, D. (2000), "Epidemiology and Prevention of Hepatitis B”, No. 1.

Ayoub, W.S. and Cohen, E. (2016), "Review Article Hepatitis B Management in the Pregnant Patient: An Update", Vol. 4 No. Table 1, pp. 241-247.

Balsano, C., Carolina, A., Cardoso, F.N., Janeiro, R. De, Carvalho-filho, R.J., Paulo, S., Schiavon, L.L., et al. (2016), "World Journal of”, Vol. 5182 No. 9, pp. 421-460.

Bartholomew, M.L. (2017), "Management of Hepatitis B Infection in Pregnancy”, Vol. 00 No. 00.

Borgia, G., Carleo, M.A., Gaeta, G.B., Gentile, I., Borgia, G., Carleo, M.A. and Gentile, I. (2012), "Hepatitis B in pregnancy", Vol. 18 No. 34, pp. 4677-4683.

Deep, A., Kumar, A., Hashmi, M. and Swaroop, S. (2016), "Maternal and Fetal Outcomes of Hepatitis B Infection in Pregnant Women", No. December.

Elefsiniotis, I., Vezali, E., Vrachatis, D., Hatzianastasiou, S., Pappas, S., Farmakidis, G., Vrioni, G., et al. 
(2015), "Post-partum reactivation of chronic hepatitis B virus infection among hepatitis B e-antigen-negative women", Vol. 21 No. 4, pp. 1261-1267.

European Association for the Study of the Liver. EASL clinical practice guidelines: Management of chronic hepatitis B virus infection. J Hepatol 2017 vol. 67 370-398.

Gyawali, C. Prakash. Gastroenterology Subspeciality Consult : Third Edition. Washington manual subspecialty consult series. 2012. P344-55.

Joshi, S.S. and Coffin, C.S. (2020), "Hepatitis B and Pregnancy: Virologic and Immunologic Characteristics", Hepatology Communications, Vol. 4 No. 2, pp. 157-171.

Pan, C.Q., Duan, Z., Dai, E., Zhang, S., Han, G., Wang, Y., Zhang, H., et al. (2016), "Tenofovir to prevent hepatitis B transmission in mothers with high viral load", New England Journal of Medicine, Vol. 374 No. 24 , pp. 2324-2334.

Tran TT. Management of hepatitis B in pregnancy: weighing the options. Cleve Clin J Med 2009; 76 Suppl 3: S25-S29.

Wilkins-haug, L. (2017), "Hepatitis B and pregnancy".

Xu, W.M., Cui, Y.T., Wang, L., Yang, H., Liang, Z.Q., Li, X.M., Zhang, S.L., et al. (2009), "Lamivudine in late pregnancy to prevent perinatal transmission of hepatitis B virus infection: A multicentre, randomized, double-blind, placebo-controlled study”, Journal of Viral Hepatitis, Vol. 16 No. 2, pp. 94-10. 